\title{
Optimal use of buffer volumes for the measurement of atmospheric gas concentration in multi-point systems
}

\author{
Alessandro Cescatti ${ }^{1}$, Barbara Marcolla ${ }^{2}$, Ignacio Goded ${ }^{1}$, and Carsten Gruening ${ }^{1}$ \\ ${ }^{1}$ European Commission, Joint Research Centre, Ispra, Italy \\ ${ }^{2}$ Fondazione Edmund Mach, IASMA Research and Innovation Centre, Sustainable Agro-ecosystems and \\ Bio-resources Department, San Michele all'Adige (TN), Italy \\ Correspondence to: Alessandro Cescatti (alessandro.cescatti@jrc.ec.europa.eu)
}

Received: 18 January 2016 - Published in Atmos. Meas. Tech. Discuss.: 15 March 2016

Revised: 9 August 2016 - Accepted: 26 August 2016 - Published: 21 September 2016

\begin{abstract}
Accurate multi-point monitoring systems are required to derive atmospheric measurements of greenhouse gas concentrations both for the calculation of surface fluxes with inversion transport models and for the estimation of non-turbulent components of the mass balance equation (i.e. advection and storage fluxes) at eddy covariance sites. When a single analyser is used to monitor multiple sampling points, the deployment of buffer volumes (BVs) along sampling lines can reduce the uncertainty due to the discrete temporal sampling of the signal. In order to optimize the use of buffer volumes we explored various set-ups by simulating their effect on time series of high-frequency $\mathrm{CO}_{2}$ concentration collected at three Fluxnet sites. Besides, we proposed a novel scheme to calculate half-hourly weighted arithmetic means from discrete point samples, accounting for the probabilistic fraction of the signal generated in the averaging period. Results show that the use of BVs with the new averaging scheme reduces the mean absolute error (MAE) up to $80 \%$ compared to a set-up without BVs and up to $60 \%$ compared to the case with BVs and a standard, non-weighted averaging scheme. The MAE of $\mathrm{CO}_{2}$ concentration measurements was observed to depend on the variability of the concentration field and on the size of $\mathrm{BVs}$, which therefore have to be carefully dimensioned. The optimal volume size depends on two main features of the instrumental set-up: the number of measurement points and the time needed to sample at one point (i.e. line purging plus sampling time). A linear and consistent relationship was observed at all sites between the sampling frequency, which summarizes the two features mentioned above, and the renewal frequency associated with the volume. Ultimately, this empirical relationship can be ap-
\end{abstract}

plied to estimate the optimal volume size according to the technical specifications of the sampling system.

\section{Introduction}

The increasing atmospheric greenhouse gas concentrations and the related global warming are raising the demand for reliable and stable observations of both greenhouse gas atmospheric concentrations and land-atmosphere fluxes. In particular, measurement of $\mathrm{CO}_{2}$ concentrations with very high precision and long-term stability are needed to derive quantitative information about the large-scale carbon fluxes using inverse modelling techniques (Andrews et al., 2014; Vardag et al., 2014). Similarly, vertical and horizontal gradients of $\mathrm{CO}_{2}$ concentration are needed to estimate non-turbulent components of the mass balance (i.e. storage and advective fluxes) at experimental sites where the land-atmosphere exchanges of mass and energy are continuously monitored by means of the eddy covariance technique (Baldocchi et al., 2001). For these purposes, in the framework of continental and global monitoring networks (e.g. ICOS, Neon, Fluxnet), accurate multi-point systems for the automatic and continuous monitoring of greenhouse gas concentrations are increasingly required and deployed. For both atmospheric concentration and land-atmosphere flux monitoring stations, the overall accuracy of measurement systems based on a single analyser that monitors multiple sample points is limited by the large uncertainty generated by the discrete temporal sampling of the signal (Heinesch et al., 2007). To limit this major source of error, Marcolla et al. (2014) proved the effec- 

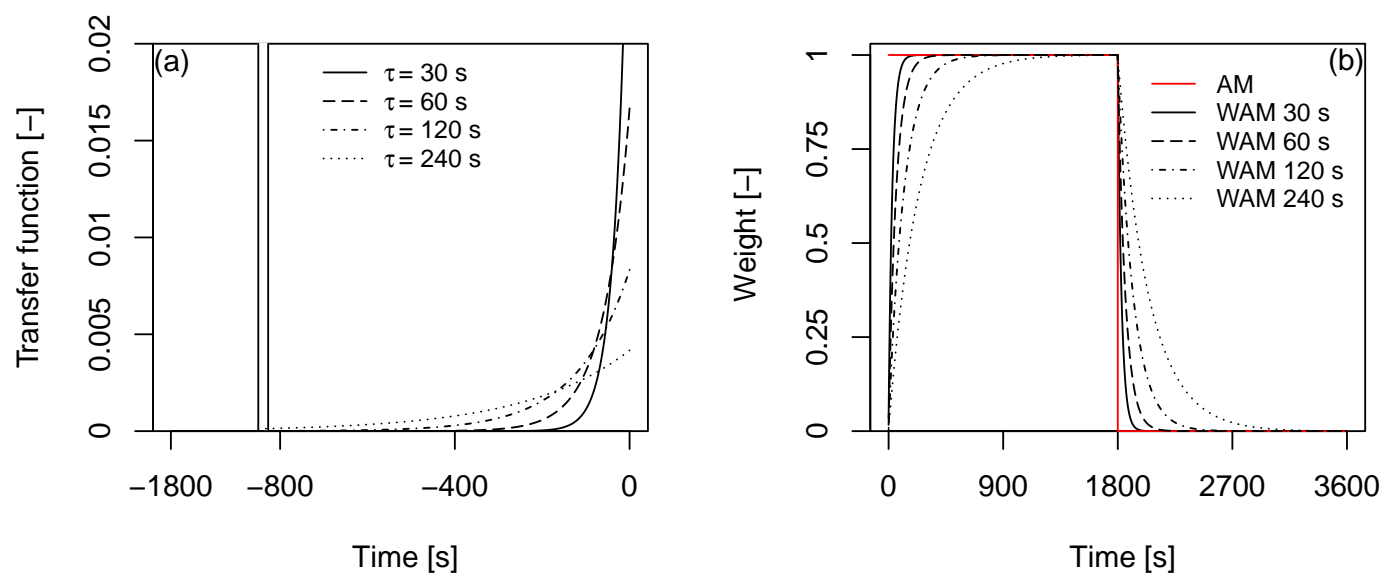

Figure 1. (a) Transfer function used to simulate the deployment of buffer volumes with four different values of residence time $\tau$ (30, 60, 120, and 240 s); (b) weight function for the calculation of half-hourly averages in the case of weighted arithmetic means (WAMs).

tiveness of buffer volumes (BVs) deployed along the sampling lines. These volumes act as a low-pass filter, supplying the analyser with a temporally mixed air flow where highfrequency fluctuations are filtered out (Griffith et al., 2008; Leuning et al., 2008). On the other hand the use of BVs introduces a temporal dependency in the measurements that has to be considered in the calculation of time averages.

To our knowledge, the complex effect of BVs in multipoint sampling systems has not been fully investigated and specific studies are required to explore the potential and limitations of their application. For this purpose, in this work we evaluate the effectiveness and optimal use of $\mathrm{BVs}$ in $\mathrm{CO}_{2}$ concentration measurements simulating the effect of idealized volumes on high-frequency time series of $\mathrm{CO}_{2}$ concentration data collected at three Fluxnet sites (IT-Isp above and below canopy, IT-Cas and IT-SR2). In order to optimize the use of BVs we propose a novel processing scheme to calculate half-hourly weighted averages from discrete point samples, by accounting for the probabilistic fraction of the signal generated in the averaging period of interest. In addition, an empirical procedure is developed to identify the optimal BV size for different system set-ups in terms of number of sampling points and schemes. The first part of the analysis focuses on observations collected above and below canopy at a mixed forest site (IT-Isp) with the objective of highlighting the effectiveness of BVs and of an alternative averaging scheme in reducing the measurement error. The second part of the analysis is based on measurements performed at three sites (IT-Isp, a coniferous forest (IT-SR2) and a rice paddy field; IT-Cas), with the objective of generalizing the findings and defining an empirical method for the optimization of the volume size on the basis of the technical specifications of the measuring system.

\section{Materials and methods}

\subsection{Study sites and instrumental set-up}

\subsubsection{IT-Isp}

The study site is located in an unmanaged forest stand in northern Italy $\left(45^{\circ} 48^{\prime} 45.72^{\prime \prime} \mathrm{N}, 8^{\circ} 38^{\prime} 1.68^{\prime \prime} \mathrm{E}\right)$ and has been operated since summer 2012. The forest is dominated by deciduous trees (Quercus robur L. (80\%), Alnus glutinosa L. (10\%), Populus alba L. (5\%) and Carpinus betulus L. (3\%)) with a mean leaf area index of $4.2 \mathrm{~m}^{2} \mathrm{~m}^{-2}$ during the growing season. The site is equipped with a self-standing tower (36 $\mathrm{m}$ high) instrumented with standard meteorological sensors (air and soil temperatures, relative humidity, air pressure, precipitation, radiation components in the shortwave and longwave), and a 3-D ultrasonic anemometer (HS-100, Gill Instruments Ltd., Lymington, UK) and an infrared gas analyser (LI-7200, Li-Cor Inc., Lincoln, Nebraska, USA) for the eddy covariance system, which acquires data at $10 \mathrm{~Hz}$ and is installed at $36 \mathrm{~m}$ above the ground ( $8 \mathrm{~m}$ above the top of the canopy). In addition, a second analyser is used for the profile system (LI-7000, acquired at $1 \mathrm{~Hz}$ ); for the specific needs of this study a closed-path analyser (LI-7000) was used to monitor the $\mathrm{CO}_{2}$ concentration at $1 \mathrm{~m}$ height, which was operated at $1 \mathrm{~Hz}$ during 2014 (DOY 107-198).

\subsubsection{IT-Cas}

The study site is located in a rice paddy field in the Po Valley northern Italy $\left(45^{\circ} 04^{\prime} 12.17^{\prime \prime} \mathrm{N}, 8^{\circ} 43^{\prime} 3.08^{\prime \prime} \mathrm{E} ; 88 \mathrm{~m}\right.$ a.s.l. $)$. The eddy covariance system, mounted at a height of $2.2 \mathrm{~m}$, consisted of a 3-D ultrasonic anemometer (HS-100, Gill Instruments Ltd., Lymington, UK) and a LI-6262 (Li-Cor Inc., Lincoln, Nebraska, USA) closed-path infrared gas analyser. The site was operated from 2006 to 2010 and is described in further details in Rossini et al. (2010) and Meijide et 
al. (2011). Data used in the present analysis were collected in 2010 (DOY 107-198).

\subsubsection{IT-SR2}

The study site is located in a maritime pine forest in central Italy (Tuscany; $43^{\circ} 43^{\prime} 43^{\prime \prime} \mathrm{N}, 10^{\circ} 17^{\prime} 13^{\prime \prime} \mathrm{E} ; 6 \mathrm{~m}$ a.s.1.). Fluxes of $\mathrm{CO}_{2}, \mathrm{H}_{2} \mathrm{O}$, and sensible heat have been continuously measured since the beginning of 2013 by means of the eddy covariance technique. Measurements are performed using an infrared gas analyser (LI-7200, operated at $10 \mathrm{~Hz}$, Li-Cor, Lincoln, NE, USA) and a 3-D sonic anemometer (HS-50, Gill instruments, Lymington, UK) installed on top of a scaffolding tower at a height of $24 \mathrm{~m}, 5 \mathrm{~m}$ above the canopy top. Additional information on the site is reported in Matteucci et al. (2015). Data used in the present analysis were collected in 2014 (DOY 107-198).

\subsection{Analytical formulation of the problem}

\subsubsection{Effects of buffer volumes on the signal}

In order to analyse the impact of BVs on the error statistics of a multi-point monitoring system, the high-frequency $\mathrm{CO}_{2}$ time series collected with the instrumental set-ups described in Sect. 2.1 were convoluted with an exponential transfer function (Eq. 1) that simulates the effect of the BVs on the signal:

$f(t)=\exp (-t / \tau)$

where the time constant $\tau=V / q$ (being $V\left(\mathrm{~m}^{3}\right)$ the volume and $q\left(\mathrm{~m}^{-3} \mathrm{~s}^{-1}\right)$ the incoming flow $)$ is the average residence time that a tiny probe volume spends in the system and is equal to the time it takes to reach $1-1 / e \sim 63.2 \%$ of its asymptotic value from a step increase (or the time to decrease in value to $1 / e \sim 36.8 \%$ from a step decrease). Its reciprocal $1 / \tau$ is referred to as renewal frequency and expresses the average number of volume renewals per unit time.

For practical reasons in this study the theoretically infinite memory of BVs is limited to half an hour. To accommodate this assumption the transfer function Eq. (1) is normalized in order to sum to one when integrated over $1800 \mathrm{~s}$, according to Eq. (2):

$f_{n}(t)=\frac{f(t)}{\int_{0}^{1800} f(\hat{t}) \mathrm{d} \hat{t}}$.

A well-mixed volume $V$ with an initial concentration $c_{0}$ will respond to an incoming flow $q$ at concentration $c_{1}$ with the following time-dependent function (Winderlich et al., 2010)

$c(t)=c_{1}+\left(c_{0}-c_{1}\right) f_{n}(t)$.

The normalized transfer function (Eq. 2) is visualized in Fig. 1a for four values of $\tau(30,60,120$, and $240 \mathrm{~s})$, which for a flow of $5 \mathrm{~L} \mathrm{~min}^{-1}$ correspond to a volume of $2.5,5,10$,

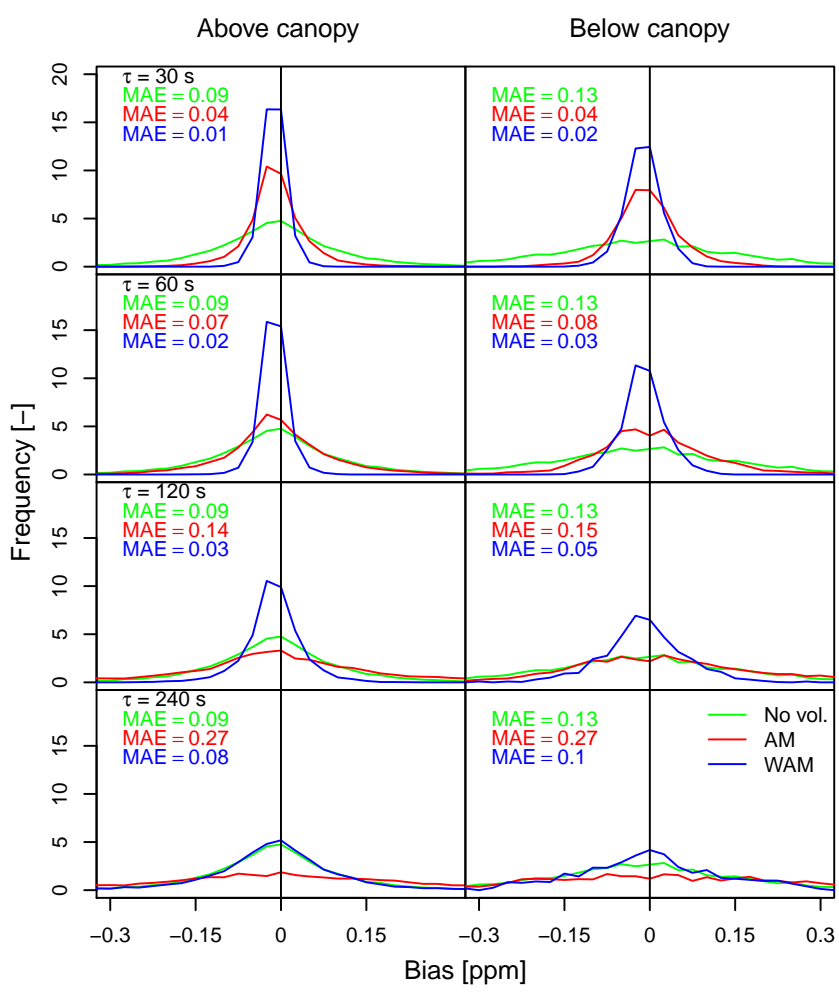

Figure 2. Frequency distributions of the bias between real and estimated $\mathrm{CO}_{2}$ concentrations above and below canopy (IT-Isp site) for four different residence times $\tau(30,60,120,240 \mathrm{~s})$ and for the three averaging schemes (NoVol: without buffer volumes, AM: with buffer volumes and simple arithmetic means, WAM: with buffer volumes and weighted means). A sampling scheme with six points and $5 \mathrm{~s}$ purging and $1 \mathrm{~s}$ reading sampling scheme was simulated.

and $20 \mathrm{~L}$, respectively. Figure 1 clearly shows that the larger the residence time, the longer is the memory effect of the $\mathrm{BVs}$ in the previous half hour. On the basis of this theoretical framework, Eq. (3) is used to calculate the filtered series of $\mathrm{CO}_{2}$ concentration from the high-resolution $\mathrm{CO}_{2}$ signal of the previous $1800 \mathrm{~s}$.

\subsubsection{Defining the optimal averaging scheme}

According to the theoretical framework presented in the previous section, the $\mathrm{CO}_{2}$ signal, filtered by the presence of a $\mathrm{BV}$ along a sampling line, is affected by the original highfrequency signal in the preceding $1800 \mathrm{~s}$. This "memory" has to be accounted for when the filtered signal is used to compute half-hourly averages from the discrete measurements of multiple sampling points.

For this purpose we propose to process the convoluted signal with a weighted averaging scheme based on the concept that the importance, and therefore the weight, of an instantaneous reading in determining the half-hourly average depends on the fraction of air build-up in the volume during the half hour of interest. According to this approach, read- 
ings at the beginning of the half hour have a low importance since they are mostly influenced by the air concentration of the previous half hour, while readings at the end of the averaging period have the highest weight. In parallel, a fraction of the signal originated in a given half-hour affects the convoluted signal in the following $1800 \mathrm{~s}$, and this information has also to be considered in the estimation of the half-hourly statistics.

To formalize this methodology we define the following weighting functions for the calculation of the mean:

$$
\begin{aligned}
& w(\hat{t})=\int_{t_{\text {start }}}^{\hat{t}} f_{n}(t) \mathrm{d} t \hat{t} \in H_{i}, \\
& w(\hat{t})=1-\int_{t_{\text {start }}}^{\hat{t}-1800} f_{n}(t) \mathrm{d} t \quad \hat{t} \in H_{i+1},
\end{aligned}
$$

where $H_{i}$ is the current half hour, $H_{i+1}$ is the following half hour, and $t_{\text {start }}$ is the starting time of current half hour. The temporal trends of the weighting functions (Eqs. 4, 5) reported in Fig. 1b show how the amount of signal transferred from one half hour to the following one depends on the residence time $\tau$ of the BV.

In order to assess the effectiveness of this new weighed averaging scheme (hereinafter referred to as WAM), halfhourly average concentrations derived from the discrete sampling for different sites and simulated set-ups have been compared with the real concentration values derived from the half-hourly block average of the high-frequency signal.

In parallel, error statistics have been computed for two alternative methodologies commonly used in multi-point monitoring systems: (i) set-up without BVs (NoVol) and halfhourly averages computed as arithmetic mean of the discrete sampling of the original signal at high frequency, (ii) set-up with BVs and half-hourly averages computed as arithmetic mean (AM) of the discrete sampling of the convoluted signal. Concerning the error sources, it needs to be pointed out that the present analysis focuses exclusively on the uncertainty due to the discrete sampling of a multi-point system and does not consider the instrumental error of the gas analyser and the associated uncertainty in high-frequency observations.

Finally, the performances of the three alternative methodologies (i.e. NoVol, AM and WAM) are compared under different architectures of the sampling system (i.e. number of points, temporal sampling scheme and $\tau$ ) with the objective of optimizing the use of buffer volumes.

\subsection{Data analysis}

The analysis of experimental data is presented in the following two sections. In the first part, the mean error introduced by the discrete temporal sampling is analysed and its dependency on the variability of the concentration field both

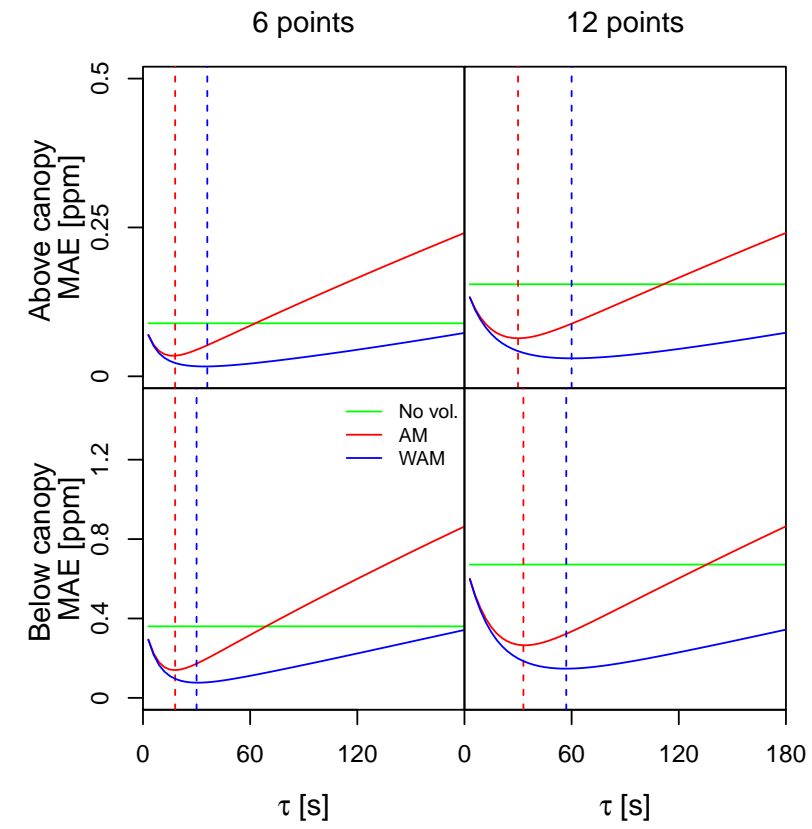

Figure 3. Dependency of the mean absolute error (MAE) on $\tau$ for the three averaging schemes (NoVol, AM, WAM) above and below canopy at the IT-Isp site. A sampling scheme with 6 and 12 points and with $5 \mathrm{~s}$ purging and $1 \mathrm{~s}$ reading was simulated. Dashed lines identify the minimum MAE and the optimum $\tau$.

in space and time is explored using data collected at IT-Isp above and below canopy; in the second section, the analysis is extended to two other sites (IT-SR2 and IT-Cas) with the aim of generalizing the findings and proposing an empirical methodology for the selection of the optimal set-up for multi-point monitoring systems.

\subsubsection{Error analysis}

From the high-frequency time series of $\mathrm{CO}_{2}$ concentration collected by the gas analysers, $1 \mathrm{~Hz}$ values were retrieved and processed to simulate the effect of different set-ups. A number of sampling points varying from 3 to 15 were simulated together with the absence or presence of BVs from 0.25 to $20 \mathrm{~L}$ with a step of $0.05 \mathrm{~L}$; switching time between points was set to $6,10,15$, and $20 \mathrm{~s}$ (assuming $5 \mathrm{~s}$ purging and $1,5,10$, and $15 \mathrm{~s}$ reading, respectively), while the flow was set at $5 \mathrm{~L} \mathrm{~min}^{-1}$. As a reference, the true half-hourly mean and standard deviation of $\mathrm{CO}_{2}$ concentration were calculated from the original $10 \mathrm{~Hz}$ time series. The frequency distribution of the half-hourly error (difference between the real concentration and the estimates retrieved for each point) was calculated for measurements collected at the IT-Isp site above and below canopy, for a scheme with six points, $5 \mathrm{~s}$ purging $+1 \mathrm{~s}$ reading cycle, four different $\tau$ (30,60, 120, and $240 \mathrm{~s}$ ), and for the three averaging schemes (NoVol, AM, WAM). Afterwards the dependency of the mean absolute error (MAE) on the variability of the concentration field repre- 


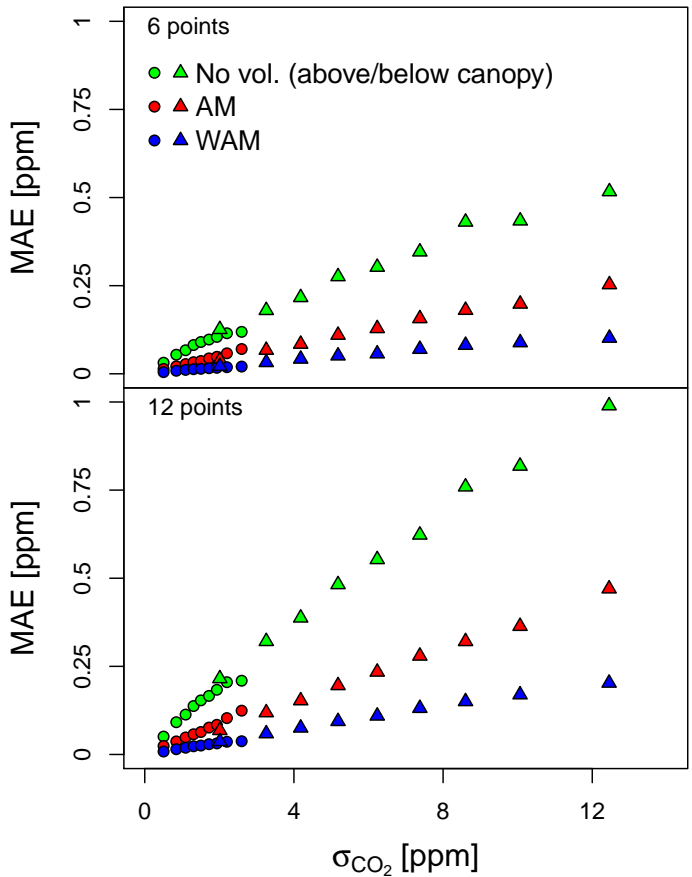

Figure 4. Dependency of the mean absolute error (MAE) on the standard deviation of half-hourly $\mathrm{CO}_{2}$ concentration. Data of the IT-Isp site collected above (dots) and below canopy (triangles) are plotted (sampling scheme as for Fig. 3). Different colours refer to the three averaging schemes (green: NoVol, red: AM, blue: WAM).

sented by the standard deviation of the $\mathrm{CO}_{2}$ concentrations and on $\tau$ was investigated for two sets of sampling points (6 and 12) and for the two different measurement heights.

\subsubsection{Optimum set-up of the monitoring system}

We define as optimal set-up the one that minimizes the MAE of $\mathrm{CO}_{2}$ concentration measurements. In an optimal set-up the size of $\mathrm{BVs}$ depends on the number of points and on the time needed to sample one point (accounting for both purging and reading time). Since these two features are summarized by the sampling frequency (defined as the number of measurement cycles per sampling point and per unit time), the empirical relationship between the optimal renewal frequency $(1 / \tau)$ of the BV and the sampling frequency has been investigated.

In the present work we focus both the theoretical framework and the analysis of the results on the concept of optimal residence time ( $\tau$ computed as the ratio of volume size and flow rate). Infinite combinations of volume size and flow rate give the same residence time and therefore can produce similar performance for the monitoring system. Given an optimal residence time, the ideal combination of BV size and flow rate has to be selected according to the technical specification of the analyser and of the other components of the sampling system (e.g. pumps, valves). In general, the use of

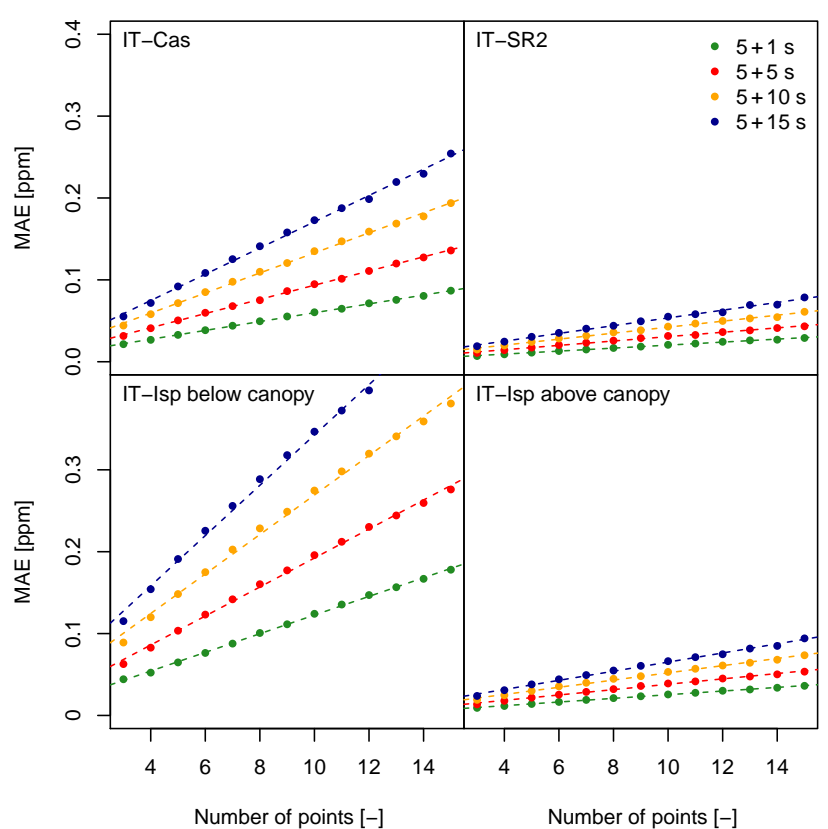

Figure 5. MAE as a function of the number of measurement points at three Fluxnet sites (IT-Isp above and below canopy, IT-Cas, ITSR2) and for four sampling schemes (5 s purging and 1, 5, 10, $15 \mathrm{~s}$ reading).

higher flow rates allows the reduction of the purging time and, therefore, increases the effective time of signal sampling.

\section{Results and discussion}

\subsection{Averaging scheme and error statistics}

The frequency distributions of the half-hourly errors were calculated for the three averaging schemes (NoVol, AM, WAM) and four residence times (30, 60, 120, and 240s) for above and below canopy at IT-Isp, simulating a set-up with six points and a $5 \mathrm{~s}$ purging $+1 \mathrm{~s}$ reading sampling scheme (Fig. 2). Error statistics highlight the advantage of using small BVs and to introduce temporal weights into the calculation of half-hourly averages from discrete point samples. Although the below canopy signal is characterized by a higher temporal variability and therefore has a larger error compared to the above canopy one, the patterns for the different averaging schemes are consistent between the two measurement heights.

The WAM approach outperforms the other methods for every value of residence time and shows the lowest MAE for a $\tau$ of $30 \mathrm{~s}$ both above and below canopy (MAE reduction of about $80 \%$ when compared to NoVol and up to $60 \%$ compared to $A M$ method). If simple arithmetic means (AM) are used for the calculation of half-hourly $\mathrm{CO}_{2}$ concentrations, the presence of $\mathrm{BVs}$ represents an advantage at low $\tau$ (up 


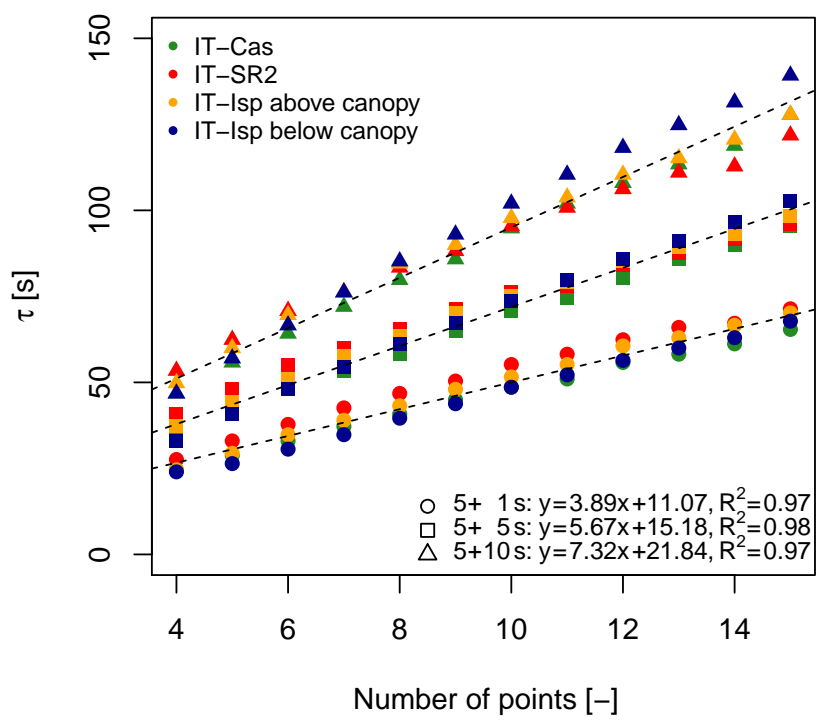

Figure 6. Optimal $\tau$ (at minimum MAE) as a function of the number of sampling points. Data are reported for four time series (IT-Isp above and below canopy, IT-Cas, IT-SR2) and for three sampling schemes ( $5 \mathrm{~s}$ purging plus $1,5,10 \mathrm{~s}$ reading) using WAM as the averaging scheme.

to $60 \mathrm{~s}$ ) and a disadvantage for higher $\tau$, when compared to the NoVol case. At $\tau=240 \mathrm{~s}$ also the WAM approach shows comparable results to the NoVol case.

The dependency of the mean absolute error on $\tau$ at IT-Isp is reported in Fig. 3 for the two cases of 6 and 12 points and a sampling scheme of $5 \mathrm{~s}$ purging $+1 \mathrm{~s}$ reading. The two averaging schemes AM and WAM both show a clear pattern of MAE, with the AM approach producing systematically larger MAEs than the WAM approach, and eventually exceeding also those of NoVol above a certain value of $\tau$. The optimal $\tau$ for the WAM approach ranges between 30 and $60 \mathrm{~s}$ depending on the number of sampling points considered and is typically about 2-fold the optimal $\tau$ for the AM averaging scheme. In addition, it is important to notice that the MAE in the case of WAM shows a lower sensitivity to $\tau$ than for AM and, therefore, proves to be a more robust methodology in the case of a sub-optimal system design. Figure 3 also shows that the optimal $\tau$ is nearly identical at the different measurement heights for both the averaging schemes.

For both above and below canopy measurements the error due to the discrete sampling of the $\mathrm{CO}_{2}$ signal depends almost linearly on the temporal variability (expressed as standard deviation) of the original signal (Fig. 4). It is worth noting that the pattern of the errors between different methods is preserved in the whole range of standard deviation of the signal. This implies that the behaviour of the different schemes is robust and consistent across a broad range of site conditions and, therefore, varies neither temporally during the day, nor spatially along the vertical profile.

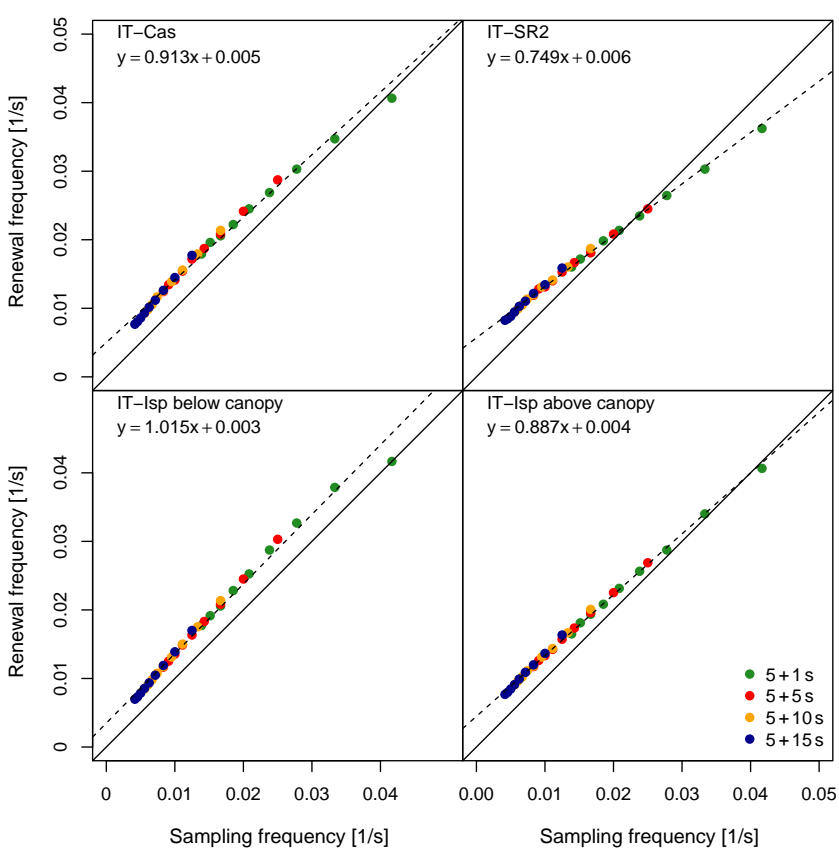

Figure 7. Optimal renewal frequency (at minimum MAE) of the buffer volume as a function of the sampling frequency for the different sites and sampling schemes. The WAM averaging scheme was used for computing half-hourly concentrations.

\subsection{Definition of the optimal set-up at multiple sites}

In this section we aim at exploring the optimal set-ups of a multi-point monitoring system at three experimental sites located in different environments (i.e. plain, coastal and hills) and characterized by different vegetation cover (i.e. crop, evergreen and deciduous forests). Given the effectiveness of the WAM averaging scheme demonstrated in Sect. 3.1, in what follows we will limit the computation of half-hourly estimates to this methodology.

Figure 5 shows the trend of MAE as a function of the number of sampling points for different sites and four alternative sampling schemes with fixed purging time $(5 \mathrm{~s})$ and variable reading time $(1,5,10,15 \mathrm{~s})$. The MAE obtained with the optimum BV is reported for each case. Results show that MAE increases with the number of sampling points for all sites and for all sampling schemes and that within each site it also increases with the length of the reading time. In fact, thanks to the temporal correlation of the convoluted signal, there is a clear advantage in shortening the reading time and switching faster between lines. The ultimate effectiveness of this strategy depends on the technical specification of the gas analyser and in particular on the time interval required to obtain a stable signal. For fast and accurate $\mathrm{CO}_{2}$ analysers a reading time of $1 \mathrm{~s}$ would be sufficient for the purpose, while for slower analysers longer reading time might be recommended.

The optimal $\tau$ and the number of points show a strong linear relationship for all sites and sampling schemes (Fig. 6). 
These optimal $\tau$ can be obtained with different combinations of BV size and flow rate, among which one can choose the more convenient for the given instrumental set-up. The observed variability between sites is significantly lower than the variability between sampling schemes, and the three linear regressions for data referring to a common sampling scheme explain about $97 \%$ of the variability (Fig. 6). The two main parameters of the monitoring systems (i.e. number of sampling points and sampling scheme) that affect the optimal $\tau$ can be combined in a single parameter, defined as the number of readings per sampling point per unit of time. This sampling frequency is closely related to the renewal frequency of the optimal BV that minimizes the MAE (Fig. 7), and this linear relationship is remarkably consistent between sites and sampling schemes. The optimal renewal frequency is generally slightly higher than the sampling frequency (in particular at low frequency), with larger slopes for sites with higher temporal variability of $\mathrm{CO}_{2}$ concentration. These relationships show that the optimal BVs are uniform for all the sampling schemes and are also rather similar among sites. Considering the low variability of the optimal residence time among sites and the low sensitivity of MAE to $\tau$ for the WAM scheme, these relationships could provide a good approximation of the optimal size of buffer volumes given a specific sampling scheme and number of sampling points.

\section{Conclusions}

In the present work, high-frequency time series of $\mathrm{CO}_{2}$ concentration collected at three Fluxnet sites were used to investigate the effectiveness and optimal use of buffer volumes in multi-point system for atmospheric measurements of greenhouse gas concentration. The use of buffer volumes can partially compensate the uncertainty due to the discrete temporal sampling of the signal. A new averaging scheme was proposed to calculate the half-hourly averages of $\mathrm{CO}_{2}$ concentration from discrete observations of multiple sampling points. This novel methodology weights the instantaneous reading of the $\mathrm{CO}_{2}$ time series on the basis of the amount of signal built up in the half hour of interest. This improves significantly the precision of the estimates $(80 \%$ reduction of MAE compared to the case without BVs and $60 \%$ compared to the case with BVs and standard averaging of the signal). The effectiveness of this averaging scheme was observed to be independent of the temporal variability of the signal and consistent in space both within and among sites. Our analysis shows that, in order to optimize the effectiveness of BVs, the residence time $\tau$ has to be carefully selected according to the technical specification of the instrumental set-up. The consistent linear relationships between the optimal $\tau$ and parameters like the number of sampling points, the temporal sampling scheme, or the sampling frequency offer an empirical method to properly dimension the BVs.
In conclusion, this analysis shows that, when the size of the volumes is optimized and the data analysis is properly performed, BVs represent a valuable technique to substantially reduce the error due to the discrete temporal sampling in multi-point monitoring systems. Even if the present work is focused on $\mathrm{CO}_{2}$, the results of the analysis and the proposed methodology are applicable to concentration measurement of any other non-reactive chemical species.

\section{Data availability}

Half hourly averages of meteorological variables and flux data of the three eddy covariance sites used in the present paper are available at http://fluxnet.fluxdata.org/data/fluxnet2015-dataset/. Highfrequency raw data are freely available upon request from the corresponding author.

Edited by: C. Brümmer

Reviewed by: three anonymous referees

\section{References}

Andrews, A. E., Kofler, J. D., Trudeau, M. E., Williams, J. C., Neff, D. H., Masarie, K. A., Chao, D. Y., Kitzis, D. R., Novelli, P. C., Zhao, C. L., Dlugokencky, E. J., Lang, P. M., Crotwell, M. J., Fischer, M. L., Parker, M. J., Lee, J. T., Baumann, D. D., Desai, A. R., Stanier, C. O., De Wekker, S. F. J., Wolfe, D. E., Munger, J. W., and Tans, P. P.: $\mathrm{CO}_{2}, \mathrm{CO}$, and $\mathrm{CH}_{4}$ measurements from tall towers in the NOAA Earth System Research Laboratory's Global Greenhouse Gas Reference Network: instrumentation, uncertainty analysis, and recommendations for future high-accuracy greenhouse gas monitoring efforts, Atmos. Meas. Tech., 7, 647-687, doi:10.5194/amt-7-647-2014, 2014.

Baldocchi, D., Falge, E., Gu, L., Olson, R., Hollinger, D., Running, S., Anthoni, P., Bernhofer, C., Davis, K., Evans, R., Fuentes, J., Goldstein, A., Katul, G., Law, B., Lee, X., Malhi, Y., Meyers, T., Munger, W., Oechel, W., Paw, K. T., Pilegaard, K., Schmid, H. P., Valentini, R., Verma, S., Vesala, T., Wilson, K., and Wofsy, S.: FLUXNET: A New Tool to Study the Temporal and Spatial Variability of Ecosystem-Scale Carbon Dioxide, Water Vapor, and Energy Flux Densities, B. Am. Meteorol. Soc., 82, 24152434, 2001.

Griffith, D. W. T., Bryant, G. R., Hsu, D., and Reisinger, A. R.: Methane Emissions from Free-Ranging Cattle: Comparison of Tracer and Integrated Horizontal Flux Techniques, J. Environ. Qual., 37, 582-591, doi:10.2134/jeq2006.0426, 2008.

Heinesch, B., Yernaux, M., and Aubinet, M.: Some methodological questions concerning advection measurements: a case study, Bound.-Lay. Meteorol., 122, 457-478, doi:10.1007/s10546-0069102-4, 2007.

Leuning, R., Zegelin, S. J., Jones, K., Keith, H., and Hughes, D.: Measurement of horizontal and vertical advection of $\mathrm{CO}_{2}$ within a forest canopy, Agr. Forest Meteorol., 148, 1777-1797, doi:10.1016/j.agrformet.2008.06.006, 2008. 
Marcolla, B., Cobbe, I., Minerbi, S., Montagnani, L., and Cescatti, a.: Methods and uncertainties in the experimental assessment of horizontal advection, Agr. Forest Meteorol., 198-199, 62-71, doi:10.1016/j.agrformet.2014.08.002, 2014.

Matteucci, M., Gruening, C., Ballarin, I. G., Seufert, G., and Cescatti, A.: Components, drivers and temporal dynamics of ecosystem respiration in a Mediterranean pine forest, Soil Biol. Biochem., 88, 224-235, doi:10.1016/j.soilbio.2015.05.017, 2015.

Meijide, A., Manca, G., Goded, I., Magliulo, V., di Tommasi, P., Seufert, G., and Cescatti, A.: Seasonal trends and environmental controls of methane emissions in a rice paddy field in Northern Italy, Biogeosciences, 8, 3809-3821, doi:10.5194/bg8-3809-2011, 2011.

Rossini, M., Meroni, M., Migliavacca, M., Manca, G., Cogliati, S., Busetto, L., Picchi, V., Cescatti, A., Seufert, G., and Colombo, R.: High resolution field spectroscopy measurements for estimating gross ecosystem production in a rice field, Agr. Forest Meteorol., 150, 1283-1296, doi:10.1016/j.agrformet.2010.05.011, 2010 .
Vardag, S. N., Hammer, S., O’Doherty, S., Spain, T. G., Wastine, B., Jordan, A., and Levin, I.: Comparisons of continuous atmospheric $\mathrm{CH}_{4}, \mathrm{CO}_{2}$ and $\mathrm{N}_{2} \mathrm{O}$ measurements - results from a travelling instrument campaign at Mace Head, Atmos. Chem. Phys., 14, 8403-8418, doi:10.5194/acp-14-8403-2014, 2014.

Winderlich, J., Chen, H., Gerbig, C., Seifert, T., Kolle, O., Lavric, J. V., Kaiser, C., Höfer, A., and Heimann, M.: Continuous lowmaintenance $\mathrm{CO}_{2} / \mathrm{CH}_{4} / \mathrm{H}_{2} \mathrm{O}$ measurements at the Zotino Tall Tower Observatory (ZOTTO) in Central Siberia, Atmos. Meas. Tech., 3, 1113-1128, doi:10.5194/amt-3-1113-2010, 2010. 